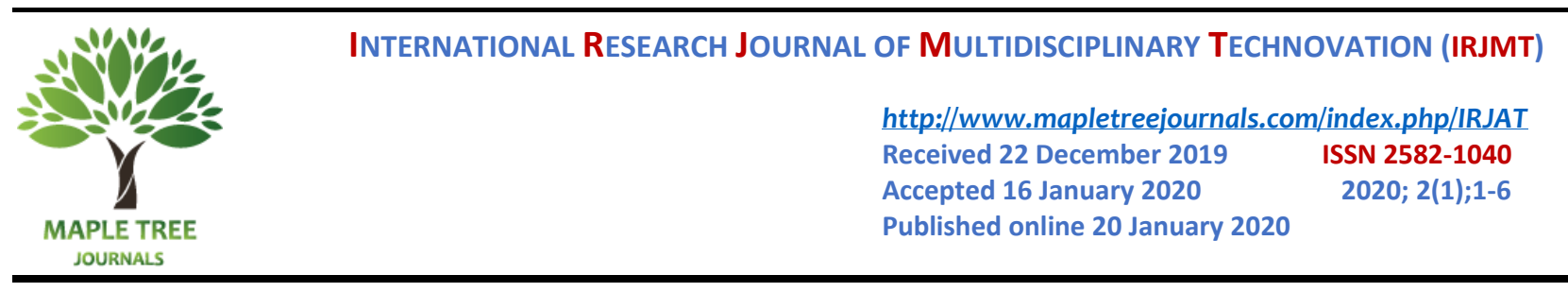

\title{
A Sitcom series to an impeccable articulation - The Role of Technology Journalism in Automobile Industries to obliterate ersatz paradigms
}

\author{
F. Paul Gregory ${ }^{* 1}$ \\ ${ }^{1}$ Department of Mechanical Engineering, PSG College of Technology, Coimbatore, INDIA \\ *Corresponding author E-Mail ID: paulgregoryresearches@zoho.com, Mobile: +91-9629431403
}

DOI: $\underline{\text { https://doi.org/10.34256/irjmt2101 }}$

\begin{abstract}
'Technology Journalism' is a rarely uttered term, but lives in day to day use, disguised in other forms of journalism. In an era where Sitcom series like 'Silicon Valley' (A HBO series which portrays events in the lives of five young men who try to start a tech company) getting quite positive responses from viewers all over the world, it is the necessity of journalists to focus more on technology journalism, rather than making fundamental reviews. Technology journalism must focus not just in reviews about new gadgets in the industry, must also play a key infotainment role. Despite several cherishing moments in this genre, the realm of technology journalism is not a bed of roses. It requires several prerequisites to efficiently carry out technology journalism. In the case of the automobile industry in India, technology journalism needs to be leveraged in a different way. With the market for automobile varying, magazines like Autocar India, WhatCar?, BiKe India and many more play a major role in taking the evolving technology to the common man. With the interests of common man revolving around lifestyle management, such magazines definitely improve the choice of customers. Hence technology journalism in the automobile industry is crucial. This article drives through fundamental technology journalism for automobile industries highlighting key aspects of the genre. This article has also thrown light into unnecessary paradigms being in practice in technology journalism and also emphasizes on pedagogy and paradigm-free journalism. This article might be ambiguous, but it is necessary to be specific and to achieve a non-agathokakological compose.
\end{abstract}

Keywords: sitcom, leverage, non-agathokakological.

\section{INTRODUCTION}

Journalism does not fall under a delimited realm. Journalism that encompasses civic journalism, political journalism, environmental journalism also holds within, this unique type of technology journalism. Technology journalism is an art of writing and reporting any advances in technology. Journalistic genres can be classified into utilitarian, informative, diversional, opinionative and interpretation [1]. From this classification, it can be interpreted that the utilitarian genre houses advertisements, promotions and recruitment calls which are required on a utility basis. Through this classification, one can identify that the informative genre can house general raw information that is needed to be conveyed to the reader. This can include incident reports, statements and explanations made by government officials and politicians, announcements, and status reports. The diversional genre house color features and recreational aspects. The opinionative genre can house grotesque cartoons, commentaries and editorial reviews which reflect the opinion of the journalist. The interpretation genre can house detailed research work on 
any particular subject. Election pre-poll surveys will eventually fall under this category. Though these genres categorize specific outcomes, the vast realm of technology journalism can fall under any of these based on the outcome. As per the American Society of Newspaper Editors, journalists must respect the rights of all others being involved in a report, and also must be accurate, responsible and must be impartial [2]. Investigative journalism is also a necessity of the hour and it is required to be linked either with academics or research or both [3].

Technology journalism for the automobile industry is unique as it requires evoking the lifestyle aspirations of an individual which will eventually result in interest or preference. The key objectives of technology journalism for the auto industry are as follows:

1. To create an auto-mania about any new addition to the industry. Be it a sports bike or a domestic car, this journalism will have to convey to target customers what it is all about.

2. To educate technical persons about the evolving automotive technology and to enable them to take a reminisce about the evolution.

3. To report the latest activities in the automobile industry, interviews, tech shows, conclaves and much more stuff related to this. This objective of technology journalism is quite common.

\section{REQUIREMENTS FOR EFFECTIVE TECHNOLOGY JOURNALISM}

Technology Journalism requires the combination of sound technical knowledge in any technological specialization and impeccable communication skills. Technology journalists are required to be articulate and precise and the choice of vocabulary must be highly decisive and is preferred to be non-colloquial. For any effective technology article, the following measures are suggested,

1. Technology journalists (TJs) must be precise and must convey their opinions as short as possible. For example, if a technology journalist is on an assignment to compose an article about the advances in Engine Valve Sourcing and Expediting, then he/she must focus on the advances rather than focusing on fundamental valve sourcing. Technology journalists must be 'specific'

2. TJs must be 'patient' as patience is the key to comprehensive exploration. TJs must aim to collect as much data as possible and this can be achieved only through waiting and allowing things to happen in its own phase. All situations will not be the same and hence patience will be the key that will uphold the moral values of every TJ in all situations.

3. Adaptability is also a requirement for TJs during Technology Journalism. Adaptability does not directly mean extreme amenability, but since TJs are required to work in teams and team cohesiveness is also an important parameter, adaptability is a major prerequisite. For example, a TJ focusing on an assignment regarding car sales must have different behaving protocols from a TJ focusing on an assignment regarding a Boiler Industry. But yet, when the same TJ does both assignments, it is mandatory for the TJ to immediately adapt to the working protocols of both variants of industry. Hence TJs must be 'adaptable'

4. In order to effectively carry out this module of journalism, a TJ must be 'inquisitive'. Possessing a character of constant learning will help in the personal building as well as for the betterment of the organization. Hence TJ must be voracious readers, great bloggers and must be effective communicators. TJs must also be capable and willing to learn multiple languages for effective communication.

5. TJs must possess fundamental knowledge of 'Business-Savvy'. Since all activities are for the automotive business, TJs must know about business activities and must be able to decipher key takeaways from various business conversations. 
6. The most important trait that a good TJ must possess is being 'Fearless'. Being bold in firing questions, precise and candor will definitely take journalism to a different level. Yes, journalism resists threats but never retreats! But it is TJs who must tread upon these and take this profession onto a different level.

7. Being digital is fun! Use of digital media to record observations, report observations is a must that every TJ must learn. Digital media is time-saving and is easy to operate more than conventional media. Hence TJs must be as 'digital' as possible.

8. To be more specific, a good technology journalism article should be 'relevant'. TJs must develop the quality of relevance and must concentrate on what is required. Journalism is a powerful profession wherein one can express his views in his own choice of language in a free non-limited environment. Journalism must not merely fill pages but must fill minds. In Technology journalism for the automobile industry, care should be taken that sufficient personnel is deployed for specific purposes to achieve this relevance. For example, if a team is specialized in analyzing the market trends of sportbikes, then that team should be motivated for achieving more on the relevant genre than training them to do car trends as well. The upper management must behave responsibly to achieve this quality.

\section{ROLE OF ENGLISH FOR TECHNOLOGY JOURNALISM FOR AUTOMOBILE INDUSTRY}

A language's ability is in how many vocabularies it can create. English is one such language that has created innumerable vocabulary making communication easier and precise. A language's true potential lies in its exotic vocabulary and making things easier. As a most reputed Hon'ble Member of the Indian Parliament Dr. Sashi Tharoor (INC) tweets "The purpose of speaking or writing is to communicate w/ precision. I choose my words because they are the best ones for the idea I want to convey, not the most obscure or rodomontade ones". As any common man could interpret from his words, precision should be the most prioritized activity in any communication. Hence Technology Journalists should be precise in their language, and in order to be precise, they would require being inquisitive not just technically, but also with the English language. The following measures will help TJs for the better use of the English language for the automobile industry.

Automotive vocabulary is like any other technical vocabulary being derived from 'morphemes'. A morpheme is the smallest distinguishable unit of vocabulary in any language and can be taken analogous to an atom in material science. In the English Language, morphemes study plays a crucial role in technical vocabulary. Every technical word has a literal meaning behind its mask and when technology journalist learns to discern this hidden meaning, and then journalists will learn the power of the English language and will learn to wield it with greater responsibility. For example, the most common term in an IC Engine is 'valve' which actually has its own roots in Latin 'valva' which means 'leaves of a door'. This means that 'valve' is an object that permits and restricts something to pass through it. This word has evolved through Middle English to be used in the Oil and Gas industry and in the automotive industry. Hence TJs when getting updated with any technical term must learn to discern its origin and must also learn to discern the literal use. With this abstract, a couple of conjectures have been invented.

\section{THE GREGORY - MORPHEME CONJECTURE}

Statement: It is apparent that there is nothing in this world to be deemed as Technical Language. All forms of vocabulary in a language are morpheme-based declensions of the literal use of the word. 
Explanation: It is observable that not just in the automobile industry, there is a paradigm that one should learn technical words to become an expert in the industry and this conjecture poses a formal objection for this paradigm. Every vocabulary used has a meaning in day to day life and the reader's inability to discern this latency has led to the discovery of so-called 'technical' words. When man created word forms, he created it to communicate what he thought. Communication is to express one's inner thoughts. Creating a separate class of 'technical' English without allowing individuals to discern the inner meaning is tantamount to discriminating individuals on the basis of the mediocre world reality and not allowing them to express one's thoughts. For example, the word 'automobile' has two components. One - 'auto' which means to do things on one's own and Two - 'Mobile' which means to move from one point to another. Hence, if one would deconstruct this simple word, it can be understood the literal meaning of 'automobile' is to move from one place to another on one's own and this distinguishes the technical functionality of automobiles and ordinary mobile. Hence it is suggested for Technology Journalists to eviscerate the so-called technical words and deconstruct them. This will give TJs greater control over their vocabulary.

\section{THE GREGORY - GLOBAL LANGUAGE CONJECTURE}

Statement: It is apparent that a single language vocabulary hypothesis is void and ineffective. An efficient language will extend its branches to all its neighborhood languages exchanging vocabulary and in continual improvement.

Explanation: It is evident that right from the days of a language's origin, a language does not remain as it is. It undergoes a series of changes and these changes will go on. This is analogous to a quasi-entropic process. This law includes even the oldest existing language in the world, 'The Great Tamil'. Readers might become ambiguous in the interpretation of this conjecture as this is the only conjecture which makes English confront this classical language. The purpose of this conjecture is not to pass a derogatory note onto any of the languages but to validate the vocabulary evolution of various languages. Right from the days of old English, English has been keen on trading vocabulary for constant improvement and this has made this language cross multiple borders and to literally rule the world. But as one would observe, the old and middle English will not be the same as modern English and has changed. This is the same phenomenon being witnessed in all other languages. Languages tend to co-exist for continual improvement. Thus it can be seen in this evolution that there cannot be a single language vocabulary commitment (though the origin can be established). For example, 'cash' in English is similar to ' $k a s u$ ' in Tamil. This can be seen as a coexisting nature and this coexisting nature is because of travelers from one region of the world to another. This conjecture is applicable to technology journalism as well. Whenever TJs experience cross-language vocabulary they must eviscerate the vocabulary to its native form. For example, let us deconstruct the so-called technical word 'crankshaft'. As a layman could interpret, anything rotating under a constrained environment can be considered as a 'shaft'. The word 'crank' can be dated back to the old English word 'cringe' which means to bend one's body and head in a fearful way to serve. Hence a shaft that is bent in a way to serve is termed as the crankshaft. This simple example will break paradigms and selfestablished strongholds in Technology Journalism for the automobile industry.

Also, TJs must also possess inquisitiveness for novels. As it is evident, every novel is unique and every writer is unique. A TJ must not just learn from other technology articles, must also possess the ability and inquisitiveness to learn from fiction and other non-fictional novels. Taking this note in a more comprehensive way, every TJ must possess the ability to understand the evolution of the language and correlate it with the so-called technical word. With the abovesaid examples, such as automobiles, crankshafts, valves, it is apparent that etymology plays a major role in technology journalism. For a longer period of time, the world has always considered 
English and Technical English to be different and kept it distinguished. This article unifies the two and asserts both are indistinguishable. Technology journalism must discern this fact and must work towards creating much more vocabulary.

\section{BREAKING STRONGHOLD PARADIGMS FOR TECHNOLOGY JOURNALISM}

Paradigms are man-made models and when this world follows a certain pattern on its own, it is not mandatory, that such paradigms need to be practiced forever. There are hot debates among journalist educators in improving the quality of journalists[4]. Amendments and dissents have strengthened our community right from its origin. Here, in technology journalism too, it is not necessary to stick to self-made paradigms. As described earlier, technology journalism is an art, which needs to be enriched through effective innovations. Here are some of the measures that are suggested to break strongholds.

Conclusions and opinion polls need not necessarily conform to worldly standards. If there is a dissent, and if there is a disagreement in general opinion, it is a prerogative of TJs to voice their opinion and also must introspect over the dissent. As the great Apostle St Paul once said, "Do not deceive yourselves. If any of you think you are wise by the standards of this age, you should become fools so that you may become wise". So, it is apparent that whatever the world proposes need not necessarily be true and with candor and is subject to amendments. Even journalism students are suggested to have a positive attitude and vast knowledge [5]. Hence, a single worldly standard can be ruled out and a concept of innovative journalism can be stepped in.

\section{THE GREGORY - STRONGHOLD PARADIGM HYPOTHESIS}

Statement: There cannot be a single protocol imposed in technology journalism as eventually, they will amend and will even cease to exist, conditionally. Eventually, every stronghold paradigm this mediocre world considers to be shall be pulled down.

Explanation: This hypothesis is an extension of St. Paul's words on conforming to the world. As it can be applied to technology journalism as well, it is apparent that there cannot even be an assumption of self-made strongholds. This hypothesis can be well explained using an automobile industry example. Consider the world's biggest auto expo is to take place in a city in Germany and leading brands exhibit their new vehicles in the expo. In such a case, a technology journalist affiliated to any journal/magazine, shall not have any prejudice or self-held standard before visiting the expo. If any new vehicle is launched, then the journalists shall examine its new features, inquire about its exotic features and must have a positive outreach mindset. A journalist who always has prejudice to correct and criticize unnecessary aspects will not be a creative one. On the other hand, as per this hypothesis, the TJ must also be open to any change, on technological backgrounds. For example, if in the expo, a new brand of bike, exhibits its new bike with 8 wheels and still claims to be a bike, then the TJ shall not jump to a conclusion that a bike should be limited to 2 wheels. TJs must be observers and analysts and not critics. Journalism is different from being critical. Instead, the TJ must examine the various possibilities in the technological expansion and must oust any conventional thought that would make one's own opinion changing. Technology is vulnerable to change and so should be technology journalism.

\section{CONCLUSION}

As the article title emphasizes, even a sitcom web series can be influential in the tech industry and hence technology journalists have the potential to change the world in a positive way literally making the world a better place to live in. Some of the key conclusions that can be inferred are as follows, 
1. Though volatile, there are certain traits for technology journalists such as patience, inquisitiveness, adaptable and many more, which enhances the quality of technology journalism.

2. The Gregory-Morpheme conjecture literally obliterates the necessity for a separate domain of technical language and stresses that a morpheme-based analysis for technology journalism will enable journalists to reach miles in their career.

3. The Gregory- Global Language conjecture poses the volatility of all language forms and also throws light into the co-existence of various languages. This conjecture based study will enable Technology journalists to eviscerate language etymology and apply that to technology journalism.

4. The Gregory - Stronghold Paradigm hypothesis literally proposes that the world will eventually change and hence there is no necessity for a single paradigm in technology journalism. This hypothesis will boost the innovative morale of technology journalists for the automobile industry.

\section{REFERENCES}

[1] J. M. de Melo and F. de Assis, Gêneros e formatos jornalísticos: um modelo classificatório, Intercom Rev. Bras. Ciências da Comun., 39 (2016) 39-56.

[2] D. Smith, Introduction to Journalism. 2013.

[3] W. Bacon, Investigative journalism in the academy-possibilities for storytelling across time and space, Pacific Journal. Rev., 17 (2011) 45-66.

[4] B. Marin, C. Viktorija, G. Jonila, and I. et al Liana, Requirements for Modern Journalism Education - The perspective of students in South East Europe, Konrad-Adenauer-Stiftung E.V., Berlin and Sofia.

[5] A. Ismail and R. Ismail, Knowledge versus experience: Indicator to good journalism practices, J. Komun. Malaysian J. Commun., 33 (2017) 142-158. 